\title{
Feature Extraction Method for Fault Diagnosis of Rotating Machinery Based on Wavelet and LLE
}

\author{
Guangtao Zhang ${ }^{1, a}$, Yuanchu Cheng ${ }^{1, b^{*}}$, Xingfang Wang ${ }^{2, c}$ and $\mathrm{Na} \mathrm{Lu}^{3, \mathrm{~d}}$ \\ ${ }^{1}$ School of Power and Mechanical Engineering, Wuhan University, Wuhan China, 430072 \\ ${ }^{2}$ School of Electric and Renewable Energy Engineering, Three Gorges Vocational College of Electric \\ Power, Yichang China, 443000 \\ ${ }^{3}$ College of Water Resources and Architectural Engineering, Northwest A\&F University, Yangling \\ China, 712100

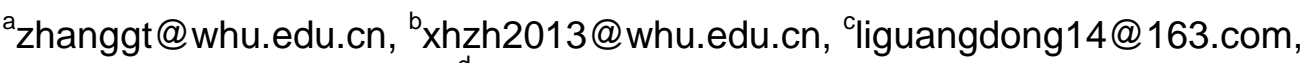 \\ dpyzhaocool@126.com
}

Keywords: Feature extraction; Rotating machinery; Fault diagnosis; Wavelet; LLE

\begin{abstract}
Feature extraction is an important procedure in the process of fault diagnosis for rotating machinery. Based on wavelet and local linear embedding (LLE), a method is proposed in this paper to extract features from vibration signals of rotating machinery. Firstly, multiple features were extracted from the original vibration signals and their wavelet decomposition coefficients to construct a high feature set. Then, to reduce the dimension of the high feature set initially, detection index (DI) was taken as an index to select several features from the extracted features. After that, LLE was employed to conduct feature fusion on the initial obtained feature set and obtain low dimension fault features for fault diagnosis of rotating machinery. To validate the proposed method, fault extraction experiment was conducted, and the result shows that the proposed method can extract better features for fault classification of rotating machinery.
\end{abstract}

\section{Introduction}

As economy developed, much more rotating machinery has becoming larger and larger, and considerable economic losses and great disaster may be caused by machinery fault. Therefore, it is of great importance to diagnosis the fault types of rotating machinery early and accurately.

The procedure of fault diagnosis for rotating machinery include signals acquisition, feature extraction and fault reorganization, among which feature extraction plays an important role on the accuracy of fault diagnosis. So far, many signal processing methods have been used to extract features from signals of machinery, such as FFT, STFT, Wigner-Ville distribution[1, 2], and wavelet [2, 3, 4] and so on. Among these methods, wavelet is developed based on FFT. But different from FFT, wavelet has advantages of good time-frequency localization properties, which enable it suitable to extract features not only related to time domain but also to frequency domain.

Local linear embedding (LLE) method is a kind of manifold learning method proposed by Roweis and Lawrence [5]. Compared with traditional linear dimension reduction method, LLE has great ability on processing of high dimension and larger-scale data. It not only has lower complexity of time and space, but also suitable to nonlinear signals feature extraction for rotating machinery. Hence, LLE has been used in domain of feature fusion $[6,7,8]$.

For rotating machinery vibration signals, plenty of features can be extracted. However, because some redundant and irrelevant features are included in these features, fault diagnosis algorithm complexity may be increased and the accuracy of the fault diagnosis result may be influenced. Feature selection can remove irrelevant features from the original feature set and feature fusion is available to reduce the redundant features from the high dimension feature set. Therefore, in this paper, multiple features were extracted from the original acquired vibration signals of rotating machinery and its wavelet coefficients firstly. Then, these features were evaluated by the detection index (DI) [9], and 
those with larger DI were selected to reduce the dimension of the original feature set initially. After that, LLE was used to fusion the selected features to form a lower feature set for fault diagnosis of rotating machinery.

\section{Theory of Wavelet and LLE}

Theory of Wavelet. Wavelet analysis is proposed by Morlet firstly in 1974. It has good time and frequency properties, hence has been used widely in industry. Wavelet transform include wavelet decomposition and wavelet reconstruction. Only wavelet decomposition was used in this paper. It can be expressed as

$$
\begin{gathered}
v_{j+1, n}=\sum_{k \in Z} h_{k-2 n} v_{j, k}, \\
w_{j+1, n}=\sum_{k \in Z} g_{k-2 n} v_{j, k},
\end{gathered}
$$

where, $\mathrm{v}$ and $\mathrm{w}$ represent the scale coefficient and wavelet coefficient, respectively; $\mathrm{h}$ and $\mathrm{g}$ represent the low pass filter and high pass filter, respectively; $j$ represents the layer number of the wavelet decomposition; $\mathrm{k}$ and $\mathrm{n}$ represent the element number of the decomposed coefficients.

Theory of LLE. The basic idea of LLE is constructing a weight vector between sample point and its neighbor points, and keeping the weight the same in low dimension domain. That is to say, the reconstruction error is minimized under the condition that the embedded map is local linear.

The process of LLE includes the following three procedures:

(1) Search $\mathrm{k}$ neighbor points of the sample point xi (l) in high dimension feature space, where $\mathrm{i}$ is the number of the sample points $\mathrm{i}=1,2, \ldots, \mathrm{N} ; 1$ represents the dimension of the sample in feature space, $1=1,2, \ldots, \mathrm{D}$.

(2) Use the obtained $\mathrm{k}$ neighbor points to reconstruct the sample point $\mathrm{xi}$ on condition that the reconstruction error is minimized.

$$
\varepsilon(\mathbf{W})=\sum_{i=1}^{N}\left\|x_{i}-\sum_{j=1}^{k} \mathbf{w}_{i j} x_{i j}\right\|^{2}
$$

where, $x i j(j=1,2, \ldots, k)$ is the $j$ th neighbor point of the sample point $x i$; wij is the weight between xi and xij, which satisfy $\sum_{j=1}^{k} w_{i j}=1 ; \mathrm{W}$ is the matrix composed by wij.

(3) Compute the output vector yi in low dimension feature space with the obtained $\mathrm{W}$ and $\mathrm{xij}(\mathrm{j}=1$, $2, \ldots, \mathrm{k})$ and Eq. 4.

$$
\varepsilon(\mathbf{Y})=\sum_{i=1}^{N}\left\|y_{i}-\sum_{j=1}^{k} \mathbf{w}_{i j} y_{i j}\right\|^{2}
$$

where, $\operatorname{yij}(j=1,2, \ldots, k)$ is the $j$ th neighbor point of the point yi, which should satisfy

$$
\left\{\begin{array}{c}
\sum_{i=1}^{N} y_{i}=0 \\
\frac{1}{N} \sum_{i=1}^{N} y_{i} y_{i}^{\mathrm{T}}=\mathbf{I}
\end{array}\right.
$$

where, I is identity matrix.

In the process of computing the optimal result of Eq.3, $\varepsilon(\mathrm{Y})$ can be expressed as 


$$
\varepsilon(\mathbf{Y})=\sum_{i=1}^{N} \sum_{j=1}^{N} M_{i j} y_{i}^{j} y_{j}=\operatorname{tr}\left(\mathbf{Y} \mathbf{M} \mathbf{Y}^{\mathrm{T}}\right),
$$

where, $\mathrm{M}$ is a $\mathrm{N} \times \mathrm{N}$ symmetric matrix which can be expressed as

$$
\mathbf{M}=(\mathbf{I}-\mathbf{W})^{\mathrm{T}}(\mathbf{I}-\mathbf{W}) .
$$

So, the $\mathrm{d}$ minimum eigenvalues of $\mathrm{M}$ is the feature fusion result $\mathrm{Y}$ with $\mathrm{d}$ dimension in lower feature space.

\section{Feature Extraction Method Based on Wavelet and LLE}

Procedure of the Feature Extraction Method Based on Wavelet and LLE. In order to obtain low dimension high sensitive features for fault diagnosis of rotating machinery, a feature extraction method based on wavelet and LLE is proposed in this paper, its procedure is illustrated as follows.

(1) The acquired signals are input into the flow chart.

(2) Decompose the signals into three layers by wavelet method and obtain the decomposition coefficients.

(3) Extract ten features [10] as shown in Table 1 from each of the original signals and the obtained decomposition coefficients, respectively, and use the obtained features to construct a high feature set.

(4) Taking DI as an evaluation index to select ten features with large value of DI from the high feature set to reduce its dimension initially.

(5) Apply LLE method to fusion the obtained ten features to remove the redundant features and obtain low dimension features for fault diagnosis of rotating machinery.

Table 1 Types of the fault features

\begin{tabular}{|c|c|}
\hline Feature & Expression \\
\hline$F_{1}$ & $\sigma / \bar{f}$ \\
\hline$F_{2}$ & $\sum_{i=1}^{N} f_{i}^{2} / \sigma^{2}$ \\
\hline$F_{3}$ & $\sum_{i=1}^{N}\left(f_{i}-\bar{f}\right)^{3} / N \sigma^{3}$ \\
\hline$F_{4}$ & $\sum_{i=1}^{N}\left(f_{i}-\bar{f}\right)^{4} / N \sigma^{4}$ \\
\hline$F_{5}$ & $\bar{f}_{p} / \bar{f}$ \\
\hline$F_{6}$ & $\bar{f}_{p} / \sigma$ \\
\hline$F_{7}$ & $\left|\sum_{i=1}^{N_{p}}\left(f_{p i}-\bar{f}_{p}\right)^{3}\right| / N_{p} \sigma_{p}^{3}$ \\
\hline$F_{8}$ & $\left|\sum_{i=1}^{N_{p}}\left(f_{p i}-\bar{f}_{p}\right)^{4}\right| / N_{p} \sigma_{p}^{4}$ \\
\hline$F_{9}$ & $\left|\sum_{i=1}^{N_{v}}\left(f_{v i}-\bar{f}_{v}\right)^{3}\right| / N_{v} \sigma_{v}^{3}$ \\
\hline$F_{10}$ & $\left|\sum_{i=1}^{N_{v}}\left(f_{v i}-\bar{f}_{v}\right)^{4}\right| / N_{v} \sigma_{v}^{4}$ \\
\hline
\end{tabular}


In Table 1, fi represents the sample point, fpi and fvi represent peak value and valley value of fi,

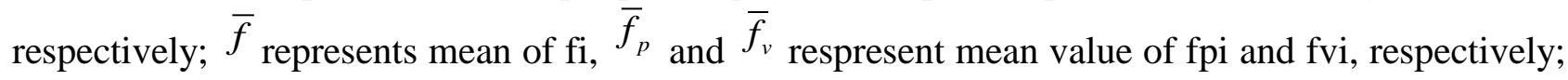
$\sigma$ is standard deviation of fi, $\sigma \mathrm{p}$ and $\sigma \mathrm{v}$ represent standard deviation of fpi and fvi, respectively.

Detection Index. In this paper, DI is adopted to evaluate the sensitivity of the obtained features and remove irrelevant ones. DI is defined as follows.

Suppose that s1 and s2 are values of a kind of feature extracted from signals acquired under different machinery conditions, respectively. Moreover, s1 and s2 obey the law of normal distribution $\mathrm{N}(\mu 1, \sigma 1)$ and $\mathrm{N}(\mu 2, \sigma 2)$, respectively, where $\mu 1>\mu 2$. Then, DI can be defined as

$$
\mathrm{DI}=\frac{\mu_{2}-\mu_{1}}{\sqrt{\sigma_{1}^{2}+\sigma_{2}^{2}}} \text { or } \mathrm{DI}=\frac{\bar{s}_{2}-\bar{s}_{1}}{\sqrt{\sigma_{1}^{2}+\sigma_{2}^{2}}}
$$

According to [9], the larger the DI is, the more sensitive the feature will be. Therefore, DI is taken as an index to evaluate the sensitivity of the features and those with large values of DI are selected.

\section{Experiment and Results}

To validate the effective of the proposed method, 20 vibration signals were acquired from a rotor experimental system under normal, unbalance, misalignment, and rub conditions, respectively. Then, 50 features in total were extracted from each of the original acquired signals and its wavelet four decomposition coefficients, and a high dimension feature set was formed. In this process, db6 wavelet is taken as the adopted wavelet. After that, DI was calculated from the high dimension feature set and the larger 10 features were selected initially to reduce the dimension of the feature set. The DI values of the selected features are shown in Table 2.

Table 2 The DI values of the selected features.

\begin{tabular}{|c|c|c|c|c|c|c|c|c|c|c|}
\hline $\mathrm{DI}$ & $S_{1}$ & $S_{2}$ & $S_{3}$ & $S_{4}$ & $S_{5}$ & $S_{6}$ & $S_{7}$ & $S_{8}$ & $S_{9}$ & $S_{10}$ \\
\hline $\mathrm{DI}_{1-2}$ & 4.13 & 6.35 & 3.33 & 1.59 & 1.95 & 0.34 & 0.57 & 1.84 & 1.66 & 2.17 \\
\hline $\mathrm{DI}_{1-3}$ & 10.30 & 6.29 & 7.14 & 6.31 & 5.28 & 4.39 & 5.45 & 2.70 & 3.46 & 1.98 \\
\hline $\mathrm{DI}_{1-4}$ & 2.08 & 1.86 & 1.70 & 0.89 & 0.54 & 3.15 & 0.22 & 0.59 & 1.89 & 0.16 \\
\hline $\mathrm{DI}_{2-3}$ & 7.67 & 5.01 & 5.60 & 7.12 & 0.76 & 3.23 & 3.93 & 3.95 & 2.50 & 2.90 \\
\hline $\mathrm{DI}_{2-4}$ & 1.28 & 1.28 & 1.21 & 1.52 & 2.30 & 2.59 & 0.57 & 0.79 & 0.70 & 2.12 \\
\hline $\mathrm{DI}_{3-4}$ & 1.20 & 0.87 & 0.59 & 1.53 & 5.82 & 0.57 & 2.34 & 2.89 & 1.72 & 1.30 \\
\hline
\end{tabular}

Considering that there still were redundant in the obtained feature set, LLE was used to fusion the selected features into low dimension features. In this process, the neighbor point number of the sample point was set to 8 , and the number of lower dimension was set to 3 . Then, the obtained 3 dimension features is illustrated in Fig. 1(a).

In order to validate the effective of the proposed method, comparison experiment when only feature selection and only feature fusion procedure was included in the process of feature extraction were also conducted, respectively. The result of the obtained lower features when only feature selection procedure was included is shown in Fig. 1(b). While the result of the obtained lower features when only feature fusion procedure was included is shown in Fig. 1(c).

From Fig. 1, it can be seen clearly that the result obtained by the proposed method can classify the four types of the signals acquired under four conditions, respectively. Whereas the results obtained when only feature selection or only feature fusion procedure was included in the process of feature extraction cannot discriminate the four conditions clearly. Therefore, by the proposed method, better features can be extracted for fault diagnosis of rotating machinery. 


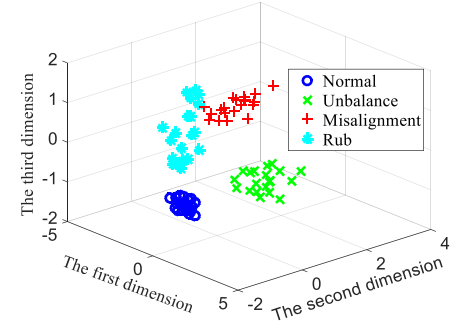

(a)

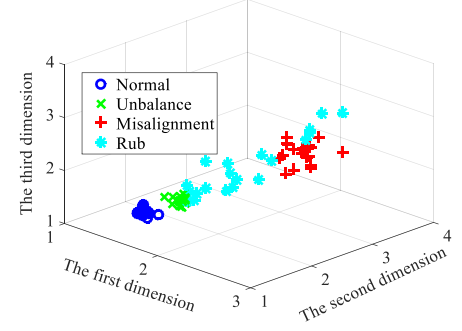

(b)

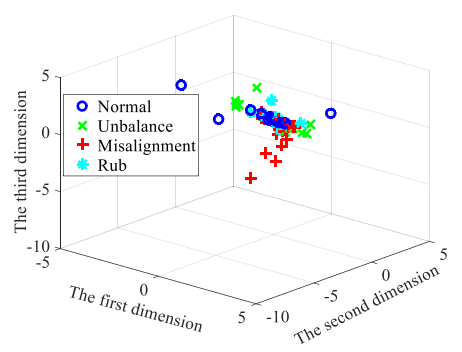

(c)

Figure. 1 Lower dimension features obtained, (a) the proposed method, (b) feature selection method, (c) feature fusion method.

\section{Conclusion}

In order to extract lower dimension high sensitive fault features for fault diagnosis of rotating machinery, a method based on wavelet and LLE was proposed in this paper. In this method, both feature selection and feature fusion procedure were included in the process of feature extraction to remove irrelevant and redundant features from the constructed high dimension feature set, respectively. Experiment result shows that, with the proposed method, better features on classification of different types of signals can be obtained. Therefore, this method can be used in the process of fault diagnosis of rotating machinery effectively.

\section{References}

[1] Wu J.D., Huang C. K., An engine fault diagnosissystem using intake manifold pressure signal and Wigner-Ville distribution technique, Expert Systems with Applications. 38(2011)536-544.

[2] Tang B.P., Liu W.Y., Song T, Wind turbine fault diagnosis based on Morlet wavelet transformation and Wigner-Ville distribution, Renewable Energy. 35(2010)2862-2866.

[3] J.Rafiee, M.A.Rafiee, P.W.Tse, Application of mother wavelet functions for automatic gear and bearing fault diagnosis, Expert Systems with Applications. 37(2010)4568-4579.

[4] Amir Hosein Zamanian, Abdolreza Ohadi, Gear fault diagnosis based on Gaussian correlation of vibrations signals and wavelet coefficients, Applied Soft Computing. 11(2011)4807-4819.

[5] Sam T. Roweis, Lawrence K. Saul, Nonlinear dimensionality reduction by locally linear embedding, Science. 290(2000)2323-2326.

[6] R. Hettiarachchi, J.F. Peters, Multi-manifold LLE learning in pattern recognition, Pattern Recognition. 48 (2015) 2947-2960

[7] Su Z, Tang B, Ma J, et al. Fault diagnosis method based on incremental enhanced supervised locally linear embedding and adaptive nearest neighbor classifier, Measurement. 48(2014)136-148.

[8] B.W. Li, Y. Zhang, Supervised locally linear embedding projection for machinery fault diagnosis, Mech. Syst. Signal Process. 25 (2011)3125-3134.

[9] Lu N, Xiao Z, Malik O P., Feature extraction using adaptive multiwavelets and synthetic detection index for rotor fault diagnosis of rotating machinery, Mechanical Systems and Signal Processing. 52 (2015)393-415.

[10] Ke Li, Peng Chen, and Huaqing Wang, Intelligent Diagnosis Method for Rotating Machinery Using Wavelet Transform and Ant Colony Optimization, IEEE Sensors Journal, 12(2012)2474-2484. 